\title{
Effects of lentivirus-mediated shRNA targeting integrin-linked kinase on oral squamous cell carcinoma in vitro and in vivo
}

\author{
LIN QUE $^{1}$, DAN ZHAO $^{3}$, XIU-FA TANG ${ }^{2}$, JI-YUAN LIU $^{2}$, XIANG-YU ZHANG ${ }^{1}$, \\ YU-HUA ZHAN $^{1}$ and LEI ZHANG ${ }^{1}$
}

\begin{abstract}
${ }^{1}$ State Key Laboratory of Oral Diseases, Sichuan University, Chengdu 610041; ${ }^{2}$ Department of Head and Neck Carcinoma, West China College of Stomatology, Sichuan University, Chengdu 610041; ${ }^{3}$ Department of Oral and Maxillofacial Surgery, The First Affiliated Hospital of Chongqing Medical University, Chongqing 400016, P.R. China
\end{abstract}

Received June 4, 2015; Accepted July 15, 2015

DOI: $10.3892 /$ or.2015.4374

\begin{abstract}
Integrin-linked kinase (ILK), a highly conserved intracellular protein of serine/threonine protein kinase activities, which is associated with the integrin and growth factor receptor signaling pathway, is involved in the regulation of cell proliferation, apoptosis, differentiation, migration and epithelial-mesenchymal transition (EMT). Findings of a previous study showed that ILK overexpression was strongly correlated with a more aggressive tumor phenotype, recurrence and poor survival for oral squamous cell carcinoma (OSCC) patients, as well as some EMT markers. In order to investigate the underlying mechanisms involved, a lentivirus-mediated short hairpin RNA (shRNA) was employed to downregulate ILK. The results showed that the knockdown of ILK inhibited cell growth, adhesion and invasion ability in vitro, and OSCC cells deficient of ILK were blocked in the S phase and underwent apoptosis. Additionally, ILK shRNA inhibited EMT by impairing the expression of Snail, Slug and Twist 2 and enhacning E-cadherin expression. ILK shRNA suppressed the phosphorylation of downstream signaling targets Akt and GSk-3 $\beta$. In addition, the knockdown of ILK inhibited tumor growth, invasion and metastasis of xenograft tumors in vivo. These results suggested that ILK is a promising therapeutic target for the treatment of OSCC.
\end{abstract}

\section{Introduction}

Oral squamous cell carcinoma (OSCC) is a common malignancy in the oral cavity, accounting for $\sim 90 \%$ of all oral tumors, with an estimated 300,000 new cases annually (1). Despite

Correspondence to: Professor Xiu-Fa Tang, Department of Head and Neck Carcinoma, West China College of Stomatology, Sichuan University, 14, (Section 3), Renmin South Road, Sichuan, Chengdu 610041, P.R. China

E-mail: tangxiufa1963@hotmail.com

Key words: oral squamous cell carcinoma, integrin-linked kinase, epithelial-mesenchymal transition, short hairpin RNA, metastasis advances in diagnosis and multimodal therapies, tumors are associated with a poor prognosis with patient 5 -year survival rates of approximately $50 \%$. The unfavorable prognosis is mainly caused by local invasion and the earlier regional lymph node metastasis that impedes complete tumor resection and leads to inevitable postoperative recurrence (2). Therefore, understanding the underlying molecular mechanisms involved in the progression of OSCC and developing a novel therapeutic strategy is imperative.

Integrin-linked kinase (ILK), a ubiquitously expressed protein with serine/threonine protein kinase activities, interacts with cytoplasmic domains of $\beta 1$ and $\beta 3$ integrins $(3,4)$. As a multifunctional adaptor protein, ILK has been regarded as a vital regulator of the phosphoinositide 3 -kinase (PI3K) signaling pathway, which activates protein kinase $B$ (PKB)/Akt activity and inhibits glycogen synthase kinase-3 (Gsk-3) activity, regulating cell proliferation, apoptosis, differentiation, migration, invasion, angiogenesis and epithelial-mesenchymal transition (EMT) $(5,6)$. EMT is a complex cell process in which cells lose their epithelial features and acquire a mesenchymal phenotype, which plays a vital role in embryogenesis, tissue remodeling, wound healing and tumor metastasis. EMT process is associated with the reduced expression of epithelial markers such as E-cadherin, and increased expression of mesenchymal markers such as C-cadherin (7-9). Overexpression of ILK has been reported in various types of cancer (10-13), promoting cell migration and invasion by inducing EMT $(12,14-17)$. Consistent with the abovementioned findings, our previous study on OSCC clinical specimens revealed that ILK facilitated the progression and metastasis of OSCC through the EMT-related upregulation of Snail and consequent aberrant expression of E- and N-cadherin (18). Knockdown of ILK has been reported to inhibit cell proliferation, migration, invasion, angiogenesis, and promotes apoptosis in different types of cancer cells, including gastric cancer, bladder cancer, lung cancer, ovarian carcinoma, and tongue cancer (19-23). However, the molecular mechanisms by which ILK contributes to the development and progression of OSCC via EMT remain to be investigated.

In the present study, a lentivirus-mediated short hairpin RNA (shRNA) targeting ILK was generated and the effects of ILK silencing on OSCC cells were investigated. The results 
revealed that the knockdown of ILK inhibited cell proliferation, invasion, blocked the cell cycle, promoted cell apoptosis and suppressed the EMT process in vitro by regulating the Akt and GSk-3 $\beta$ signaling pathways. Knockdown of ILK also suppressed tumor growth, invasion and metastasis in nude mice xenograft models. In summary, the results indicated that ILK is imperative in the development and progression of OSCC and has the potential to be an efficient therapeutic target for OSCC treatment.

\section{Materials and methods}

Cell line and culture. The human OSCC BCaCD885 cell line was obtained from the State Key Laboratory of Oral Diseases, Sichuan University (Chengdu, China) and cultured in Dulbecco's modified Eagle's medium (DMEM; Gibco-Life Technologies, Grand Island, NY, USA) supplemented with $15 \%$ fetal bovine serum (FBS; Hyclone, Logan, UT, USA), $1 \%$ penicillin/streptomycin at $37^{\circ} \mathrm{C}$ in a humidified atmosphere with $5 \% \mathrm{CO}_{2}$. The cells were routinely passaged every 2-3 days by trypsinization (Gibco-Life Technologies). The cells at the logarithmic growth phase were used in the present study.

Construction of lentiviral vectors expressing ILK-specific shRNA and transfection $(24,25)$. The ILK-specific target sequence was selected according to online siRNA tools utilized from Invitrogen (http://www.invitrogen.com/rnai) and the GenBank (accession no. NM 004517.2). Double-stranded DNA containing the interference sequences were synthesized according to the manufacturer's instructions (sequence: 5'-CGA AGC TCA ACG AGA ATC A-3') (GeneChem, Shanghai, China), and the negative control shRNA targeted a random and non-specific sequence with no homology to the human genome. The sequences were cloned into the pGCSIL-green fluorescent protein (GFP; GeneChem) to generate the lentiviral vectors, and then transfected into BCaCD885 cells. On the day of infection, the culture medium was replaced with the appropriately titered viral supernatant and incubated at $37^{\circ} \mathrm{C}$ for $12 \mathrm{~h}$. After 3 days of transfection, the number of GFP-positive cells was measured, and shRNA ILK-silencing efficiency was determined using western blotting. The titers were averaged to $8 \times 10^{9} \mathrm{TU} / \mathrm{ml}$. The lentivirus containing the human ILK shRNA-expressing cassette was used as a positive control and designated as the $\mathrm{KD}$ group. The empty vector was used as a negative control, designated as the $\mathrm{NC}$ group, and the non-transfected as the $\mathrm{CON}$ group.

RNA isolation and quantitative PCR. Total RNA was prepared using TRIzol reagent (Invitrogen-Life Technologies, Carlsbad, CA, USA) and cDNA was synthesized using the PrimeScript ${ }^{\circledR}$ RT reagent kit (Takara, Dalian, China). ILK mRNA expression was evaluated quantitatively by RT-qPCR with SYBR ${ }^{\circledR}$ Premix Ex Taq ${ }^{\mathrm{TM}}$ (Takara) and the ABI PRISM 7900HT Real-Time PCR system (ABI, Vernon, CA, USA). The primers were synthesized (Sangon, China) and the sequences used were: ILK forward, 5'-ATG GAA CCC TGA ACA AAC ACT-3' and reverse, 5'-AGC ACA TTT GGA TGC GAG AAA-3'; and GAPDH forward, 5'-GCT GTC CCT GTA CGC CTC TG-3' and reverse, 5'-TGC CGA TGG TGA TGA CCT GG-3'. GAPDH served as an internal control. The thermocy- cler conditions used were: preheating at $95^{\circ} \mathrm{C}$ for $30 \mathrm{sec}, 95^{\circ} \mathrm{C}$ for $5 \mathrm{~min}, 30$ cycles of $10 \mathrm{sec}$ at $95^{\circ} \mathrm{C}, 20 \mathrm{sec}$ at $60^{\circ} \mathrm{C}, 30 \mathrm{sec}$ at $72^{\circ} \mathrm{C}$. The relative amount of PCR product was evaluated as the comparative threshold cycle of the sample divided by that of GAPDH $\left(2^{-\Delta \Delta C t}\right)(26)$. Experiments were carried out in triplicate and repeated three times.

Protein extraction and western blot analysis. The cells were washed with ice-cold PBS twice and treated with cell lysis buffer $(50 \mathrm{mmol} / \mathrm{l}$ Tris, $\mathrm{pH} 7.8,150 \mathrm{mmol} / \mathrm{l} \mathrm{NaCl}, 1 \%$ Nonidet-40), then centrifuged at $175 \mathrm{x}$ g for $10 \mathrm{~min}$, and the supernatants were collected and stored at $-80^{\circ} \mathrm{C}$. Protein concentration was measured using the bicinchoninic acid assay (BCA Protein Assay kit; Thermo Fisher Scientific lnc., Rockford, IL USA). Equal amounts (50 $\mu \mathrm{g}$ ) of protein were heated at $95^{\circ} \mathrm{C}$ for $5 \mathrm{~min}$, separated by $8-12 \%$ SDS-PAGE gel, and electrotransferred onto polyvinylidene fluoride (PVDF) membranes (Bio-Rad, Hercules, CA, USA). The PVDF membranes were then blocked with 5\% skim milk in Tris-buffered saline and incubated overnight at $4^{\circ} \mathrm{C}$ with monoclonal primary rabbit antibody of anti-human ILK, cyclin A, cyclin B1, cyclin D1, CD31, Snail (Cell Signaling Technology, Danvers, MA, USA), Akt, p-Akt (Ser473), Gsk-3 $\beta$ and p-Gsk-3 $\beta$ (Ser9) (Bioworld Technology, St. Louis Park, MN, USA), E-cadherin, Slug, Twist2 and polyclonal primary mouse antibody of anti-human caspase-3, MMP-9 (1:1,000 dilution; Beijing Zhongshan Biotechnology, Beijing, China) and washed four times with PBS-T. Subsequently, the membranes were incubated with HRP-conjugated secondary antibodies of goat anti-rabbit or goat anti-mouse IgG (1:1,000 dilution) at room temperature for $1 \mathrm{~h}$ and washed four times with PBS-T. The signal was then visualized with an enhanced chemiluminescence assay according to the manufacturer's instructions (LumiGLO Peroxidase Chemiluminescent Substrate kit; KPL, Gaithersburg, MD, USA). The amounts of proteins were quantified by densitometry and GAPDH of the same sample served as an internal control.

Colony formation assay. The effect of ILK silencing on the colony formation of $\mathrm{BCaCD} 885$ cells was analyzed using the colony formation assay. Approximately $3 \times 10^{2}$ cells were plated in $60-\mathrm{mm}$ dishes and cultured in $10 \%$ FBS DMEM at $37^{\circ} \mathrm{C}$, $5 \% \mathrm{CO}_{2}$ for 2 weeks. The cell colonies were washed twice with PBS, fixed with methanol for $5 \mathrm{~min}$ and stained with crystal violet for $20 \mathrm{~min}$, and then washed twice using $\mathrm{ddH}_{2} \mathrm{O}$. A colony was defined as aggregates consisting of $\geq 50$ cells, and manually counted under a microscope (Leica DM LB2, Wetzlar, Germany). The experiments were performed in triplicate and repeated three times.

Cell cycle analysis. BCaCD885 cells were collected and washed with ice-cold PBS, fixed with 70\% ethanol for $24 \mathrm{~h}$, then centrifuged at $600 \mathrm{x}$ g for $5 \mathrm{~min}$, and resuspended in propidium iodide (PI; Sigma, St. Louis, MO, USA) supplemented with RNase A and incubated at $37^{\circ} \mathrm{C}$ in the dark for $30 \mathrm{~min}$, then analyzed using a flow cytometer (Beckman Coulter, Brea, CA, USA) to measure the cell cycle distribution. The relative proportions of the cells in the individual cell-cycle phase fraction were determined from the flow cytometry data. The experiments were performed in triplicate and repeated three times. 
Cell apoptosis analysis. After the cells were harvested, $5 \times 10^{5}$ cells were rinsed twice with $\mathrm{PBS}$, and centrifuged at $600 \mathrm{x} \mathrm{g}$ for $5 \mathrm{~min}$ and resuspended in $500 \mu \mathrm{l}$ binding buffer. The cells were then incubated for $15 \mathrm{~min}$ with $1 \mu \mathrm{l}$ of Annexin V-PE and $5 \mu 1$ 7-AAD for $15 \mathrm{~min}$ at room temperature in the dark according to the manufacturer's instructions (Annexin V-PE/7-AAD Apoptosis Detection kit; Keygentec, China). Cell apoptosis was analyzed using flow cytometry (Beckman Coulter). The experiments were carried out in triplicate and repeated three times.

Transwell invasion assay. After reaching $80 \%$ confluence, the $\mathrm{BCaCD} 885$ cells were digested with $0.05 \%$ trypsin and resuspended at $5 \times 10^{4}$ cells $/ 100 \mu \mathrm{l}$. Prior to cell incubation, the cells (300 $\mu \mathrm{l}$ ) and $200 \mu \mathrm{l}$ of serum-free DMEM were added to the in the inner chamber, and DMEM medium (500 $\mu \mathrm{l})$ with $10 \%$ FBS to the lower chamber, between which the Matrigel gel (BD Biosciences, Billerica, MA, USA) was placed on the surface of the filtration membrane of the Transwell micropore with an aperture of $8 \mu \mathrm{m}$. Following incubation at $37^{\circ} \mathrm{C}$ for $24 \mathrm{~h}$, the non-invading cells in the inner chamber and the extracellular Matrigel were gently removed and washed with PBS. The number of invasive cells that migrated through the gel to the lower surface of the membrane were stained with $1 \%$ crystal violet for $20 \mathrm{~min}$ and images were captured under a phase contrast microscope in five random fields. Results were presented as the mean percentage of the control (control OD at $562 \mathrm{~nm}$ assigned as 100\%). Each experiment was performed in triplicate and repeated three times.

Cell adhesion assays. Prior to cell cultivation, $50 \mu 1$ of extracellular matrix gel (ECM gel; Sigma) mixed with serum-free DMEM (1:1) and $50 \mu \mathrm{l}$ serum-free DMEM containing $10 \mathrm{~g} / \mathrm{l}$ bovine serum albumin (BSA; Sigma) were added to 96 -well plates and incubated at $37^{\circ} \mathrm{C}$ for $30 \mathrm{~min}$. BCaCD885 cells were trypsinized and resuspended at $5 \times 10^{4}$ cells $/ 100 \mu \mathrm{l}$ in DMEM medium. Cells $(100 \mu \mathrm{l})$ were added to the 96 -well plate and cultured for $4 \mathrm{~h}$. After incubation, the non-adherent cells were removed by PBS washing, and adherent cells were fixed and stained with $1 \%$ crystal violet for $20 \mathrm{~min}$ at $37^{\circ} \mathrm{C}$. Absorbances at $590 \mathrm{~nm}$ were determined using a microplate reader (Bio-Rad).

Animals and tumor xenograft treatment model. Fifteen 6-week-old BALB/C-nu/nu mice were purchased from Sichuan University Experimental Animal Center (Sichuan, China), bred and held under specific pathogen-free conditions. The mice were allowed access to food and water ad libitum prior to surgery under optimal conditions (12-h light/dark with humidity at $60 \pm 5 \%, 22 \pm 3^{\circ} \mathrm{C}$ ). The protocol was approved by the Ethics Committee of the State Key Laboratory of Oral Diseases, Sichuan University. Animal procedures were performed according to the internationally accepted ethical guidelines.

Single-cell suspension of $100 \mu \mathrm{l}$ containing $2 \times 10^{6}$ BCaCD885 cells was injected subcutaneously into the skin of the two armpits for each mouse. When tumors formed on day 7 after inoculation, the mice were randomly divided into three treatment groups, $n=5$ mice per group. Different treatments were performed on the three groups over four consecutive days. For group 1, one side was injected with $1 \times 10^{7}$ TU $(50 \mu 1)$ lentivirus-encoded shRNA targeting ILK
(KD) and the other side was injected with $50 \mu 1$ sterilizing saline (CON). Mice in group 2 were injected on one side with $\mathrm{KD}$ and the other side with $1 \times 10^{7} \mathrm{TU}(50 \mu \mathrm{l})$ negative control lentivirus (empty vector, NC). Mice in group 3 were injected on one side with NC and the other side with CON. The time of tumor formation was recorded, and 28 days after injection, the mice were sacrificed. The xenograft tumors were excised and weighed, fixed in $10 \%$ buffered formalin at room temperature for pathology analysis, and the other tumor tissues were immediately placed in liquid nitrogen for the frozen section of immunofluorescence assay.

Histology and immunohistochemistry. The tumor tissue, lymph node and vital organs harvested from mice xenograft were fixed in $10 \%$ buffered formalin and embedded in paraffin, and 5- $\mu \mathrm{m}$ sections were stained with hematoxylin and eosin. The microvessels were counted from 10 different fields under the microscope at a magnification of $\mathrm{x} 200$, corresponding to areas with the highest density of vessels.

After being fixed and embedded, the samples were dewaxed twice in xylene for $15 \mathrm{~min}$ and rehydrated in gradient concentration of ethanol (approximately 10-15 ml) at room temperature. The sections were treated with $3 \%$ hydrogen peroxide for $30 \mathrm{~min}$ at room temperature and rinsed three times with PBS to block non-specific antibodies. The samples were incubated in primary antibodies of rabbit antihuman ILK (1:100 dilution; Cell Signaling Technology), Snail (1:200 dilution) and E-cadherin (1:100 dilution) (both from Abcam, Cambridge, MA, USA), caspase-3 (1:100 dilution), Ki67 (1:100 dilution) and MMP-9 (1:100 dilution) (Beijing Zhongshan Biotechnology) separately at $37^{\circ} \mathrm{C}$ for $60 \mathrm{~min}$ and incubated overnight at $4^{\circ} \mathrm{C}$. The sections were subsequently washed three times with PBS, and incubated in secondary antibodies for $40 \mathrm{~min}$ at $37^{\circ} \mathrm{C}$ using DAB staining (DAB Horseradish Peroxidase Color Development kit; Beijing Zhongshan Biotechnology). The sections were then counterstained with hematoxylin, dehydrated and mounted. To detect tumor angiogenesis, the sections were incubated in the monoclonal primary mouse anti-human CD31 (1:100 dilution; BD Biosciences) overnight at $4{ }^{\circ} \mathrm{C}$, and the remainder of the procedure was performed as mentioned above. Observations were performed under an Olympus multifunction microscope (Olympus, Tokyo, Japan) and fluorescence microscope (Leica DM LB2). Each section was examined independently by two pathologists and five fields of view were randomly selected under an optical microscope at magnification of x 200 .

Statistical analysis. Data were presented as the means \pm SD. Differences between groups were analyzed by one-way ANOVA. Statistical analysis was performed using the SPSS 13.0 software package (SPSS Inc., Chicago, IL, USA). $\mathrm{P}<0.05$ was considered to indicate statistical significance.

\section{Results}

Observation of transfection efficiency and identification of ILK expression. The transfection efficiency was determined under a fluorescence microscope. A large number of cells exhibited bright green fluorescence, which represented high transfection efficiency, while no green fluorescence was 
A

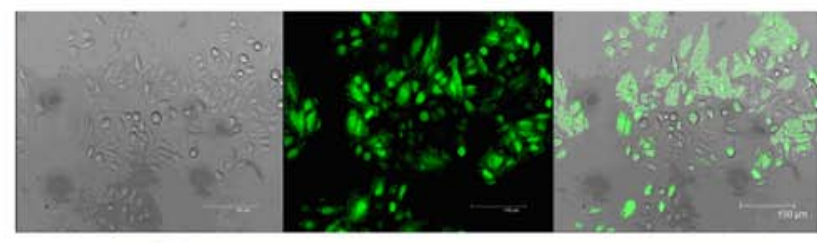

B

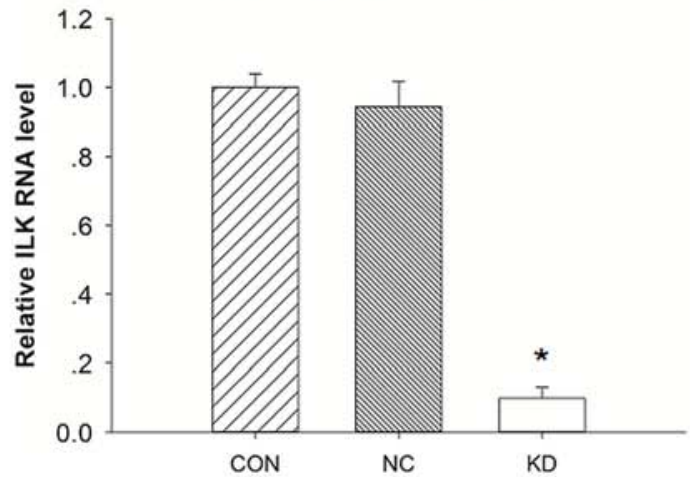

C

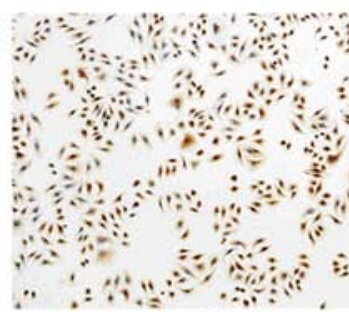

CON

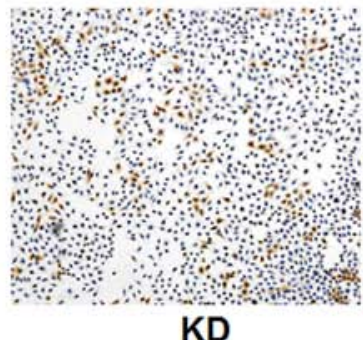

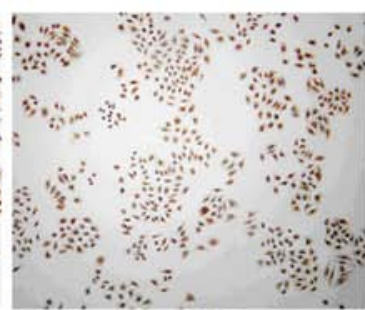

NC

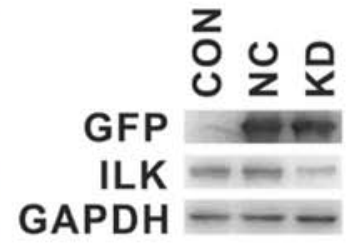

Figure 1. Observation of transfection efficiency. (A) The observation of transfection efficiency for cells under fluorescence microscope (magnification, $\mathrm{x} 200$ ). The image shows that BCaCD885 shILK cells exhibit high transfection efficiency with bright green fluorescence. (B) Detection of LIK mRNA expression by qPCR (data were presented as $2^{-\Delta \Delta C t}$, bars $\pm \mathrm{SD},{ }^{*} \mathrm{P}<0.05$ ). CON represents BCaCD885 cells, NC represents $\mathrm{BCaCD} 885$ vector cells and KD represents BCaCD885 ILK knockdown cells. The results show that mRNA expression of ILK was significantly downregulated in the KD group compared with the NC and CON groups, with a statistically significant result. (C) Immunofluorescence observation and western blot assay of ILK expressions in the three groups (magnification, x100). The results show that ILK was significantly weaker in the KD group compared with the remaining groups. ILK, integrin-linked kinase.

detected in non-transfected cells (Fig. 1A). The transfected cells were isolated by G418 selection, and then cultured and identified using qPCR, western blotting and an immunofluorescence assay. The mRNA expression of ILK was significantly downregulated in BCaCD885 ILK knockdown cells (KD group) compared with the $\mathrm{BCaCD} 885$ vector cells (NC group) and $\mathrm{BCaCD} 885$ cells (CON group), and the results were statistically significant $(\mathrm{P}<0.05$, Fig. 1B). By contrast, there was no significant difference on ILK mRNA expression between $\mathrm{CON}$ and NC cells.

Immunofluorescence images showed that ILK was significantly weaker in KD cells compared with the control cells (Fig. 1C). The protein expression of ILK was significantly reduced by $80.0 \%$ in the KD cells compared with the NC cells.

Effects of ILK knockdown on cell cycle and cell apoptosis. The cell cycle profile was analyzed by flow cytometry (Fig. 2A). The data revealed that the percentage of KD cells blocked in the $\mathrm{S}$ phase $(48.67 \pm 3.36 \%)$ was significantly higher compared with the CON cells $(24.36 \pm 2.75 \%)$ and NC cells $(33.03 \pm 5.86 \%)$, respectively $(\mathrm{P}<0.05$, Fig. $2 \mathrm{~B})$. The results suggested that the knockdown of BCaCD885 ILK cells were arrested at the restriction point of the cell cycle and failed to enter mitosis, leading to cell apoptosis.

The cell apoptotic rate was evaluated by flow cytometry. As shown in Fig. 2B, the results showed that the early apoptotic cells $\left(\mathrm{APC}^{+}, \mathrm{PI}^{-}, 32.2 \%\right)$ and late apoptotic cells $\left(\mathrm{APC}^{+}\right.$, $\left.\mathrm{PI}^{+}, 27.9 \%\right)$ in the KD group were significantly higher than the control groups $(\mathrm{P}<0.01)$, which indicated that the inhibition of cell proliferation may be caused by apoptotic cell death.

Knockdown of ILK suppressed cell adhesion, invasion and cell proliferation of BCaCD885 cells. We examined the effects of ILK silencing on the cell adhesion and invasion of BCaCD885 cells. The cell adhesion assay showed that the number of KD cells adhering to the extracellular cell matrix gel was markedly decreased compared to the remaining groups $(\mathrm{P}<0.01)$, and there was no significant difference between the $\mathrm{NC}$ and $\mathrm{CON}$ groups (P>0.05, Fig. 3B). Furthermore, to determine the effect of ILK knockdown on cell invasion ability, a Transwell assay was applied. As shown in Fig. 3A, the cells cultured in Transwell chambers were allowed to migrate through the $8-\mu \mathrm{m}$ pores of membranes coated with Matrigel. The penetrating cells in the KD group were fewer than those in the control groups $(\mathrm{P}<0.05$, Fig. 3C), which was consistent with the adhesion assays. These results demonstrated that the knockdown of ILK suppressed the abilities of adhesion and invasion of $\mathrm{BCaCD} 885$ cells.

The colony formation assay was applied for the detection of cell proliferation, and the results showed that the KD cells had a significantly lower cell proliferation rate than the remaining cells $(\mathrm{P}<0.01)$. However, no significant difference was observed between the $\mathrm{NC}$ and $\mathrm{CON}$ groups ( $\mathrm{P}>0.05$, Fig. 3D).

Effects of downregulating ILK on the expression of proteins associated with cell proliferation, invasion, apoptosis and $E M T$. To determine the underlying mechanism of downregulating ILK on cell proliferation, invasion, apoptosis and EMT, western blot analysis was applied to detect the expression of the corresponding proteins. Images showed that the expression of cyclin A, cyclin B1 and cdc2, key proteins regulating the cell cycle from $S$ phase to G2/M phase, were significantly downregulated in KD cells compared with the control cells (Fig. 4A). The expression of cyclin D1, which was involved in regulating the cell cycle from $\mathrm{G} 1$ to $\mathrm{S}$ phase, was only slightly downregulated (Fig. 4A). Fig. 4A also showed that the expression of MMP-9, which refers to the ability of cell invasion, was inhibited in ILK knockdown cells compared with the control cells. Notably, the expression of key regulating proteins of EMT, including Snail, Twist2 and Slug, were also reduced in 
A
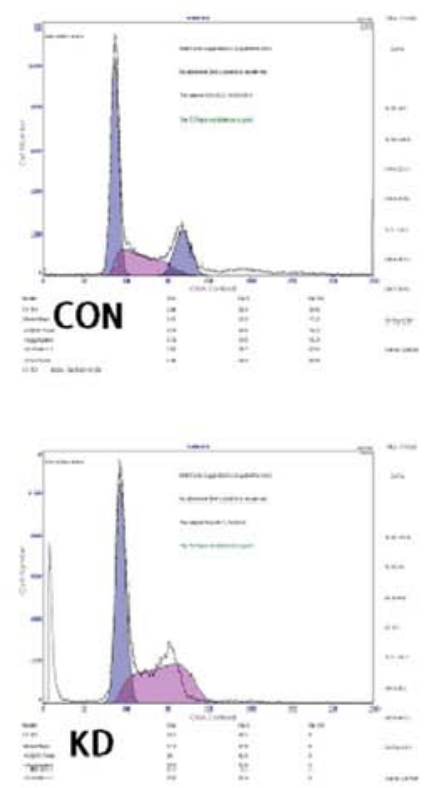

B
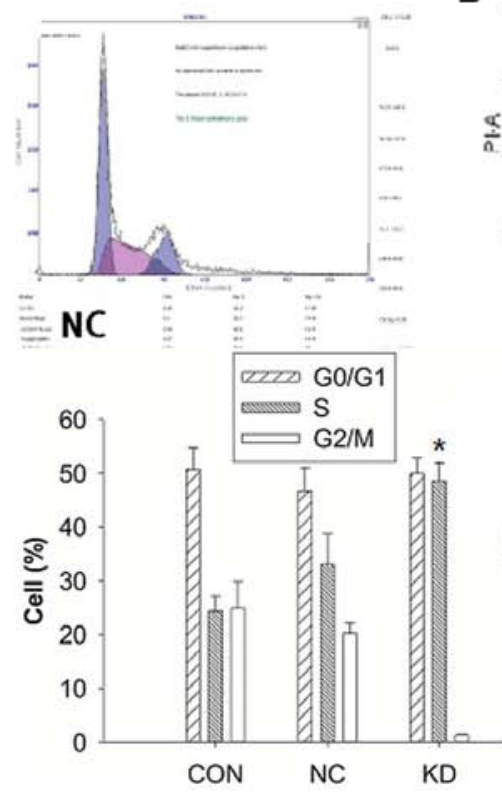
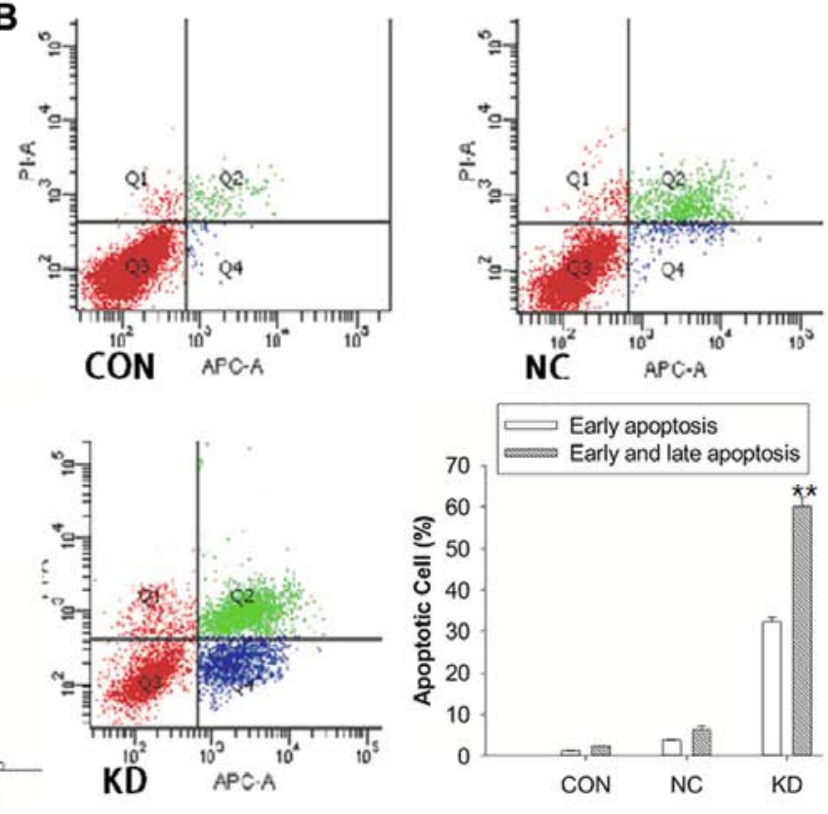

Figure 2. Effects of ILK knockdown on cell cycle and apoptosis. (A) Cell cycle was analyzed by flow cytometry using PI staining. Representative images of cell cycle distribution. Cell cycle analysis showed that the cell cycle distribution of KD cells was significantly increased in the $\mathrm{S}$ phase and decreased in the G2/M phase, compared with the CON and NC cells, respectively. (B) Flow cytometry images of cell apoptosis. Early apoptotic cells were observed by $\mathrm{APC}^{+}, \mathrm{PI}^{-}$and late apoptotic cells $\mathrm{APC}^{+}, \mathrm{PI}^{+}$. The results show that early and late apoptotic cells in $\mathrm{KD}$ cells were significantly higher than the remaining cells $\left({ }^{* *} \mathrm{P}<0.01\right)$. ILK, integrin-linked kinase; PI, propidium iodide.
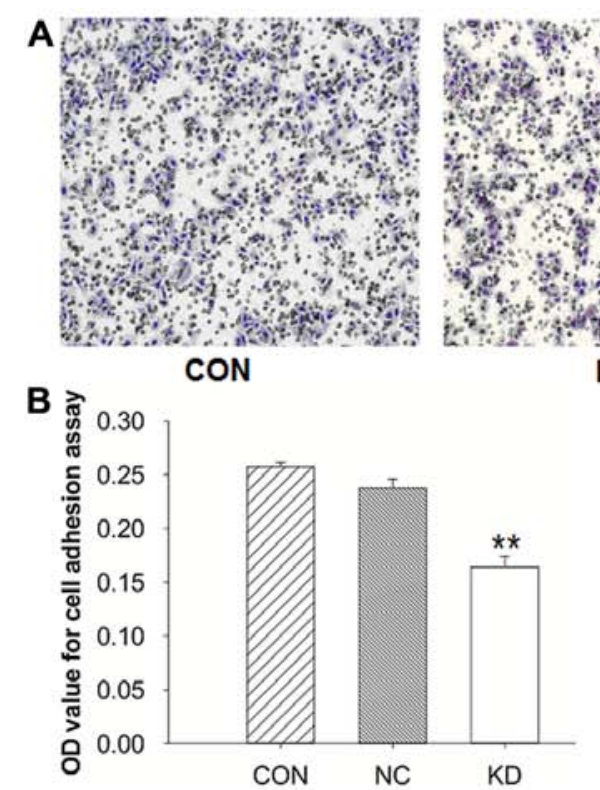
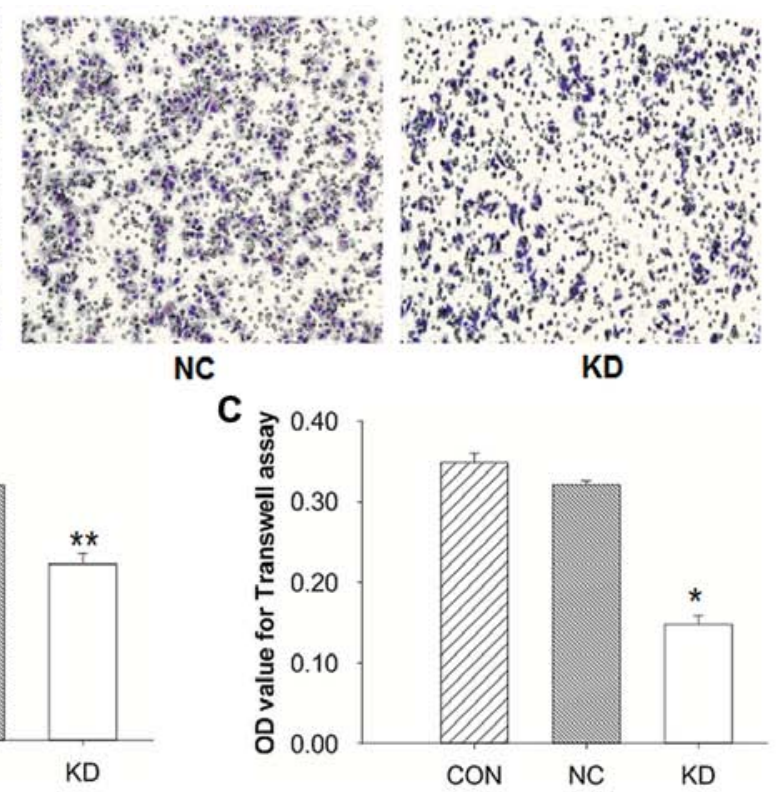

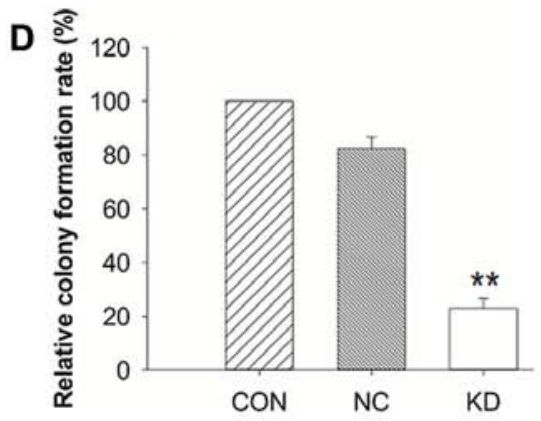

Figure 3. Effects of downregulating ILK on cell adhesion, invasion and proliferation. (A) The detection of cell invasive ability using the Transwell assay (magnification, x100). Cells $\left(5 \times 10^{4}\right)$ migrated through Matrigel-coated Transwell (8- $\mu \mathrm{m}$ pores) for $24 \mathrm{~h}$ and were counted by image analysis. (B) Data of the Transwell assay showed that cell invasion was markedly suppressed in the KD group compared with the control groups (** $\mathrm{P}<0.01)$. (C) Cell adhesion assay showed the ability of cells to adhere to the extracellular cell matrix gel. The adhesion ability of the KD group was significantly weakened compared to the control groups $\left({ }^{*} \mathrm{P}<0.05\right)$. (D) Detection of cell proliferation using a colony formation assay. The data show that KD cells had a significantly lower cell proliferation compared with the control cells ( $\left.{ }^{* *} \mathrm{P}<0.01\right)$. ILK, integrin-linked kinase.

KD cells. A protein band of cleaved Snail was observed in ILK knockdown cells (Fig. 4A). At the same time, the expression of E-cadherin was slightly upregulated in the KD cells. An increase of caspase- 3 protein was detected in KD cells, indicating that ILK may promote cell apoptosis by upregulating caspase-3. The findings suggested that downregulating ILK suppressed EMT and the invasiveness of OSCC cells.

Effects of ILK knockdown on the expression of downstream signaling target molecules. To examine the ILK downstream 


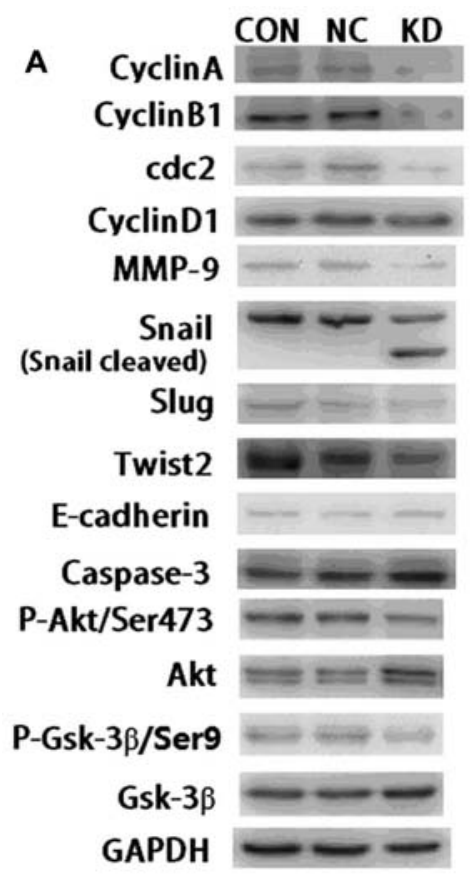

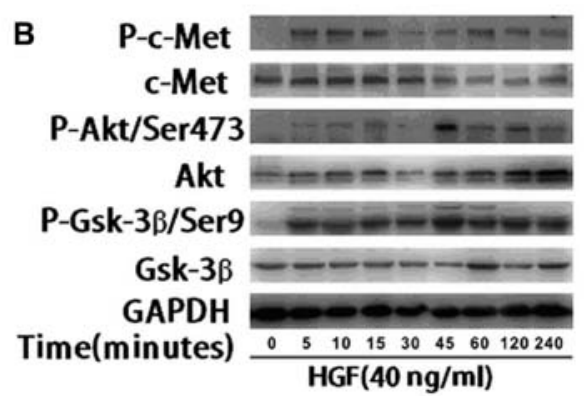

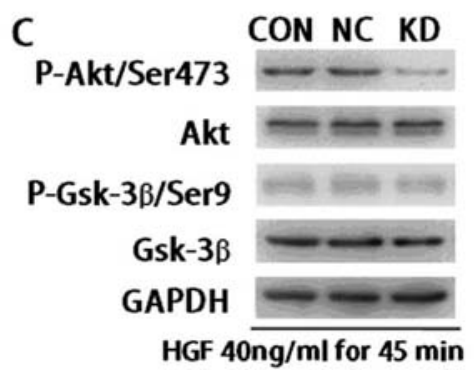

Figure 4. Effects of ILK knockdown on the protein expression of genes associated with cell proliferation, invasion, apoptosis, EMT and downstream signaling molecules in BCaCD885 cells. (A) Western blot analysis of relative proteins in KD, NC and CON cells. Cyclin A, cyclin B1, cyclin D1, cdc2, MMP-9, Snail, Twist2, Slug, E-cadherin and caspase-3 proteins were examined by western blot analysis, using GAPDH protein as the control. The results show that the protein expression of E-cadherin and caspase-3 was increased, while the protein expression of cyclin A, cyclin B1, cyclin D1, cdc2, MMP-9, Snail, Twist2, Slug, p-Akt and p-Gsk-3 $\beta$ was suppressed in the KD group compared with the control groups. (B) BCaCD885 cells were treated with $40 \mathrm{ng} / \mathrm{ml} \mathrm{HGF}$ and the expression of Akt, Gsk-3 $\beta$, c-Met and their phosphorylations were detected by western blot analysis. The expression of phosphorylation of the HGF receptor c-Met was

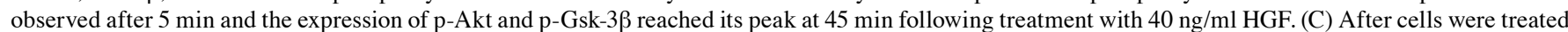
with $40 \mathrm{ng} / \mathrm{ml} \mathrm{HGF}$ for $45 \mathrm{~min}$, the expression of p-Akt and p-Gsk-3 $\beta$ was evidently weaker in ILK knockdown cells compared with the remaining cells. ILK, integrin-linked kinase; Gsk-3, glycogen synthase kinase-3; EMT, epithelial-mesenchymal transition; HGF, hepatocyte growth factor.

targets and molecular mechanisms of the effects that ILK knockdown exerted on BCaCD885 cells as mentioned above, we investigated the protein levels of Akt, Gsk-3 $\beta$ and their phosphorylated formation. Western blot analysis showed that expression of p-Akt (Ser473) and p-Gsk-3 $\beta$ (Ser9) were inhibited in ILK knockdown cells compared with the control cells (Fig. A). However, the expression of Akt and Gsk-3 $\beta$ showed no difference in the three groups (Fig. 4A).

To determine the effects of ILK knockdown on the phosphorylation of Akt and Gsk-3 $\beta$ induced by exogenous growth factors, we used hepatocyte growth factor (HGF) as an intervention, which was capable of activating PI3K and was present in the tumor microenvironment. The phosphorylation levels of Akt and Gsk-3 $\beta$ were detected by western blot analysis. The results showed that $5 \mathrm{~min}$ after BCaCD885 cells were treated with HGF at $40 \mathrm{ng} / \mathrm{ml}$, c-Met, the phosphorylation of HGF receptor was observed. After $45 \mathrm{~min}$, the phosphorylation level of Akt and Gsk-3 $\beta$ reached its peak (Fig. 4B). The phosphorylation level of Akt and Gsk-3 $\beta$ in ILK knockdown cells was not as significant as in the control cells. These results suggested that ILK was able to inhibit HGF-induced Akt and Gsk-3 $\beta$ phosphorylation to a certain extent (Fig. 4C).

Knockdown of ILK inhibits tumor growth and metastasis of $B A L B / C$ nude mouse xenograft model. To investigate whether ILK shRNA inhibited the growth of tumors in a nude mouse xenograft model, $2 \times 10^{6}$ cells in $0.1 \mathrm{ml}$ PBS were implanted subcutaneously into the armpits of BALB/C nude mice. The growth of mouse xenograft tumors was signifi- cantly inhibited in the KD group compared with the control groups (Fig. 5A). Mice were sacrificed and the tumors were collected and stained with H\&E (magnification, x100) and immunohistochemistry (Fig. 5D). The average tumor sizes were $1,032 \pm 182 \mathrm{~mm}^{3}$ in the KD group, $3,021 \pm 606 \mathrm{~mm}^{3}$ in the $\mathrm{NC}$ group and $2,835 \pm 571 \mathrm{~mm}^{3}$ in the CON group (Fig. 5B), and the average tumor weights were $302 \pm 188 \mathrm{mg}$ in the KD group, $817 \pm 237 \mathrm{mg}$ in the $\mathrm{NC}$ group and $857 \pm 235 \mathrm{mg}$ in the CON group (Fig. 5C). The inhibiting rate of tumor growth was 55.56 or $53.57 \%$, respectively. Statistical analysis revealed significant differences between the KD group and the remaining groups $(\mathrm{P}<0.05)$.

Mice from the KD group also showed a significant inhibition of the spontaneous lymph node or distant metastasis, in which no metastasis was observed, whereas lymph node metastasis (Fig. 6A) was detected in $2 / 10$ of the mice injected with negative control lentivirus and in $3 / 10$ of the mice injected sterilizing saline. One case of mediastinum metastasis (Fig. 6B) was detected in the CON group. Fig. 6C and D show the representative images of immunohistochemical staining of cytokeratin and H\&E sections (magnification, x100).

Immunohistochemical staining of CD31 was applied to detect the correlation between new blood vessel formation and tumor growth. Data revealed that the KD group showed a low CD31 expression and apparent inhibition of angiogenesis in the tumor tissue, while intense CD31 expression and a higher number of vessels were observed in the control groups (Fig. 6E). The KD group showed less vessels compared with the control groups $(\mathrm{P}<0.01$, Fig. $6 \mathrm{~F})$. 

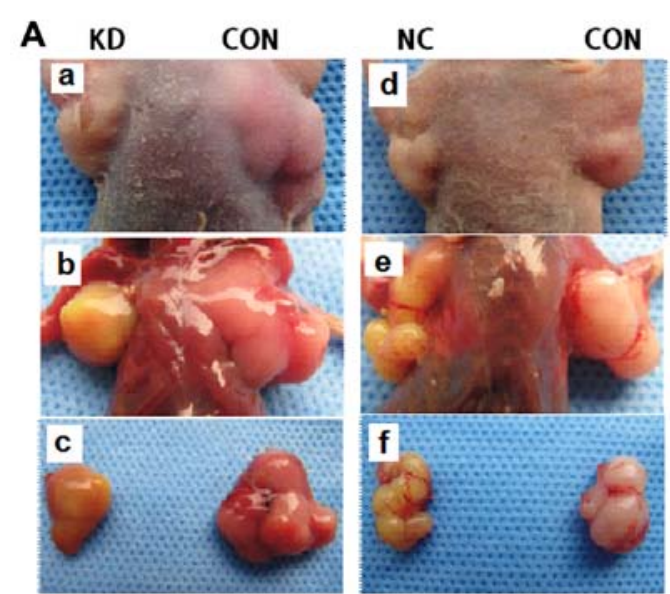

D

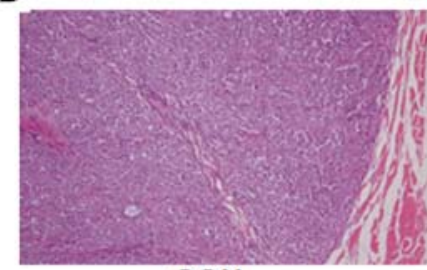

CON

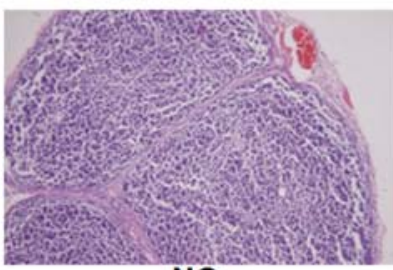

NC

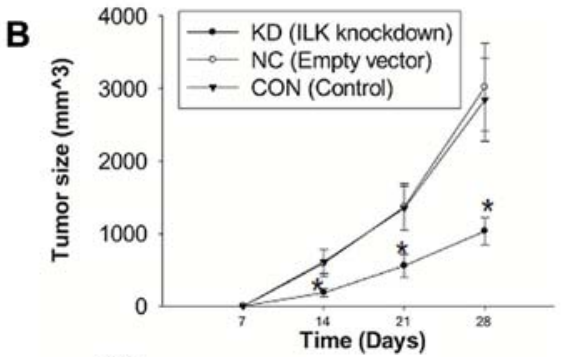

C

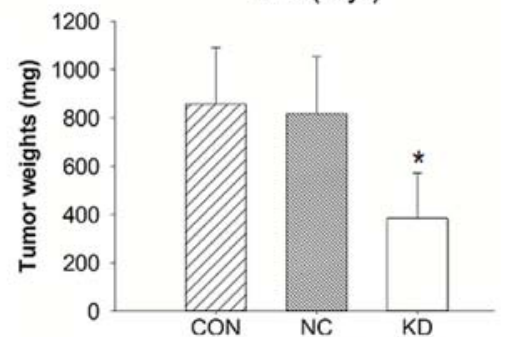

Figure 5. Local injection of lentivirus-delivered ILK shRNA inhibits tumor growth of the BALB/C nude mouse xenograft model. (A) Representative images of BALB/C nude mice with xenograft tumors. (a-c) Left sides are images of xenograft tumors injected with lentivirus-encoded shRNA targeting ILK (KD). (d-f) Left sides are images of xenograft tumors injected with the negative control lentivirus (NC). (B) Tumor sizes were closely observed and recorded every 7 days, and presented as a growth curve using the mean volumes \pm SD. (C) Tumor weight analysis, represented as the means of 10 tumors from individual mice in each group $\left({ }^{*} \mathrm{P}<0.05\right)$. (D) H\&E staining of xenograft tumors in each group (magnification, $\left.\mathrm{x} 100\right)$. ILK, integrin-linked kinase.

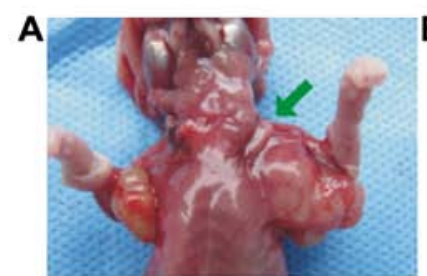

Lymph node metastasis

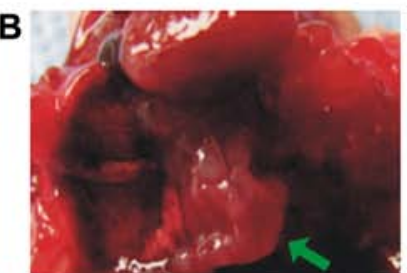

Mediastinum metastasis
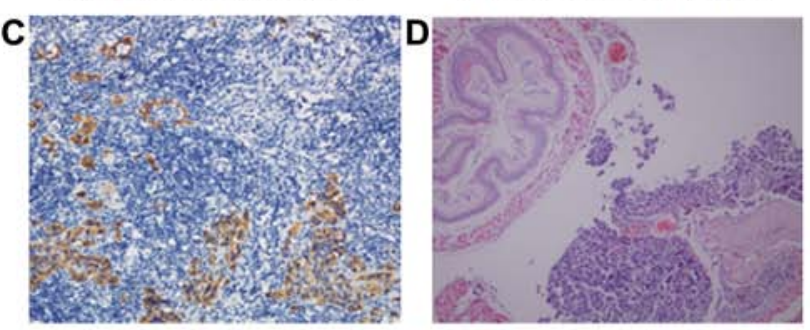

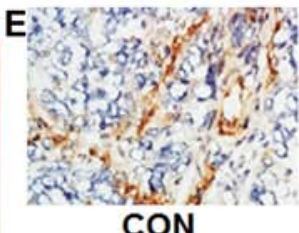

CON

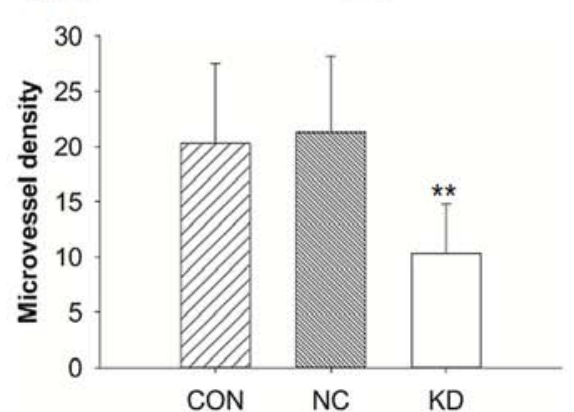

Figure 6. Downregulation of ILK inhibits the metastasis and angiogenesis of the mouse xenograft model. (A) Typical images of lymph node metastasis from mouse xenograft tumor injected with sterilizing saline. The green arrow shows the lymph node metastasis. (B) The image of mediastinum metastasis from the mouse xenograft tumor injected with empty vector, and the green arrow show the mediastinum metastasis. (C) Immunohistochemical staining with an antibody against cytokeratin antigen (magnification, x100). (D) Representative image of lymph node metastasis on H\&E sections (magnification, x100). (E) Immunohistochemical staining with an antibody against CD31 antigen of vascular endothelial cells, showing that numerous microvessels were evident among the tumors of the BALB/C nude mice in the control groups, whereas there were few microvessels or were absent in tumors of the KD group (magnification, $\mathrm{x} 200$ ). (F) The microvessel density values are presented as means \pm SD. The analysis indicated that the ILK knockdown group showed markedly fewer vessels compared with the control groups ( $\left.{ }^{* *} \mathrm{P}<0.01\right)$. ILK, integrin-linked kinase.

Immunohistochemical assays were performed to confirm ILK expression and its impact on some of its downstream signaling target molecules associated with EMT in xenograft tumors (Fig. 7). The results indicated that the KD group had an apparent decreased expression of ILK, Ki67, MMP-9, Snail as well as a higher caspase-3 and E-cadherin expression in 


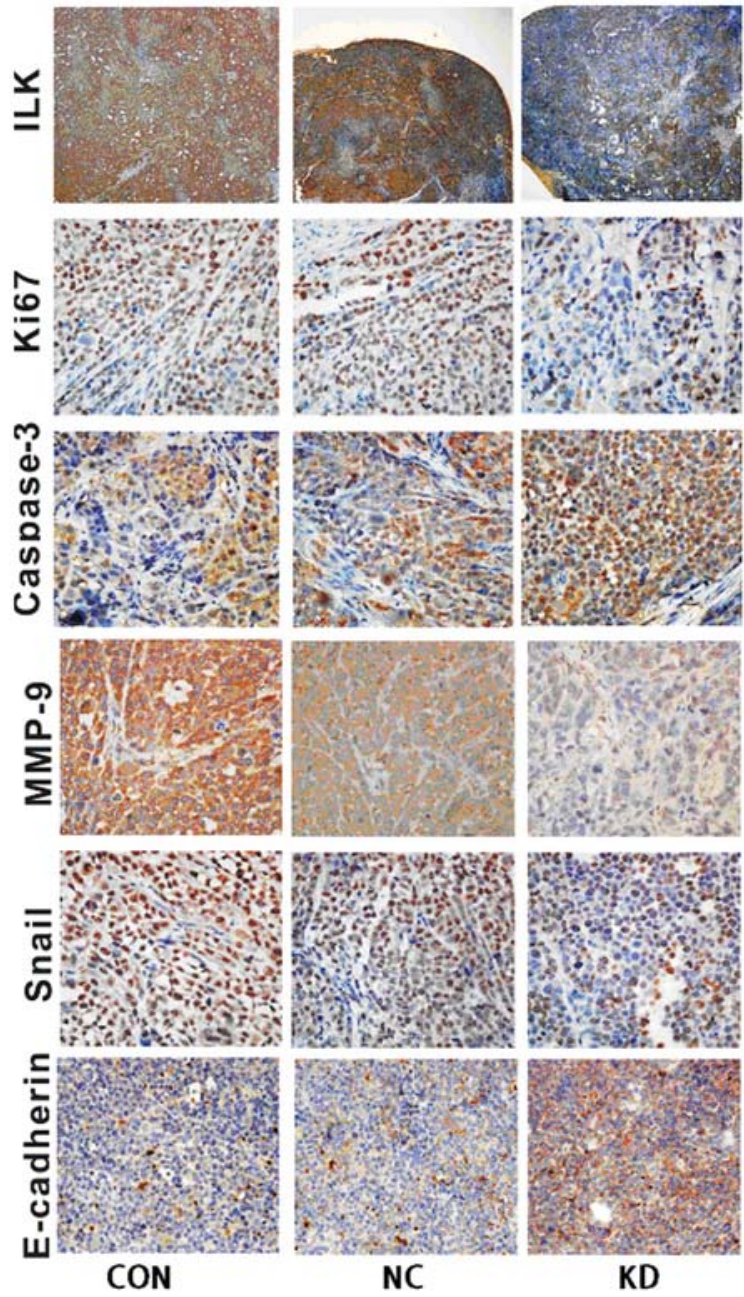

Figure 7. Effects of ILK knockdown on the expression of ILK proteins and the impact on its downstream signaling target molecules associated with EMT in xenograft tumors. Immunohistochemical staining assay of antibodies of rabbit anti-human ILK, Ki67, Snail, MMP-9, caspase-3 and E-cadherin, respectively, the nuclei were counterstained using hematoxylin (ILK, magnification $x 40$; others, magnification $x 400$ ). Typical images demonstrated that the KD group had a strong positive staining for E-cadherin and caspase-3, but weak positive staining for ILK, Ki67, MMP-9 and Snail compared with the control groups. ILK, integrin-linked kinase; EMT, epithelial-mesenchymal transition.

tumor tissue, compared with the control groups (Fig. 7). The results were in concordance with assays in vitro and supported the suggestion that the knockdown of ILK suppressed OSCC tumor growth, metastasis and EMT in vitro and in vivo.

\section{Discussion}

OSCC is a lethal epithelial cancer occurring in the oral cavity. The disease is associated with a poor prognosis resulting from its local invasion and early regional lymph node metastases (27). Previously, by analyzing OSCC clinical specimens, we revealed that ILK overexpression was associated with the progression and metastasis of OSCC, possibly through EMT-related upregulation of Snail and the consequent aberrant expression of E- and N-cadherin (15). The underlying mechanisms of invasion and metastasis of OSCC remain to be elucidated, although previous findings have (28) demonstrated that EMT may be of great importance.
ILK is a widely expressed multifunctional intracellular effector of cell-matrix interactions that play a vital role in the regulation of numerous cell processes including EMT. EMT or EMT-like phenomena are considered a prerequisite for metastasis, in certain cases (29). The functional loss of E-cadherin and presence of $\mathrm{N}$-cadherin are regarded as characteristics of the EMT program (30). The downregulation of E-cadherin, an epithelial cell marker and a potent suppressor of tumor cell invasion and metastasis, is mediated through several repressors, such as Snail, Slug and Twist (31).

In the present study, we have demonstrated that the knockdown of ILK inhibited the EMT process with increased E-cadherin expression and decreased N-cadherin expression in vitro and in vivo, which is the hallmark of EMT (30). Transcriptional repression is a major mechanisms leading to the downregulation of E-cadherin, which is suppressed by repressors such as Snail, Slug, Twist, ZEB1 and vimentin $(32,33)$. Our findings showed that the knockdown of ILK inhibited EMT-associated transcription factors such as Snail, Slug and Twist2, as well as MMP-9 expression in vitro and in vivo. We also demonstrated that downregulation of ILK inhibited cell proliferation, adhesion and invasion, arrested the cell cycle, and promoted cell apoptosis in vitro. Radeva et al (34) reported that ILK signaling pathways increased the expression of cyclin D1, activation of cyclin-dependent kinase 4 and cyclin E-associated kinases, promoting the cell cycle from the $\mathrm{G} 1$ to the $\mathrm{S}$ phase. Inhibition of ILK induced cell cycle arrest at the G1 phase $(35,36)$. However, the results showed that the percentage of KD cells blocked in the S phase $(48.67 \pm 3.36 \%)$ was significantly higher, as compared with the control groups, suggesting the cell cycle was arrested at the restriction point and failed to enter mitosis, leading to cell apoptosis. Cell apoptosis analysis revealed that the early apoptotic cells $\left(\mathrm{APC}^{+}, \mathrm{PI}^{-}, 32.2 \%\right)$ and late apoptotic cells $\left(\mathrm{APC}^{+}, \mathrm{PI}^{+}, 27.9 \%\right)$ in the KD group were significantly increased compared with the control groups. Western blotting images demonstrated that the protein expression of cyclin A, cyclin B1 and Cdc2, key proteins regulating cell cycle from the $S$ phase to $G 2 / M$ phase, decreased significantly, while the cyclin D1 protein expression slightly inhibited the KD group. Studies by Fielding et al (37-39) indicated that, ILK played a critical and unexpected role in the organization of centrosomal protein complexes during mitotic spindle assembly and DNA segregation. Thus, the knockdown of ILK resulted in mitosis failure and the cell cycle was arrested at the $\mathrm{S}$ phase. These findings suggest that downregulation of ILK suppressed the development and progression of OSCC cells.

Furthermore, the knockdown of ILK inhibited the phosphorylation of its downstream targets Akt on Ser473 and Gsk-3 $\beta$ on Ser9, respectively, and Gsk-3 $\beta$ downregulated Snail phosphorylation on Ser246. The knockdown of ILK inhibited the phosphorylation of Akt, and inhibited Akt activity (40). Thus, ILK and Akt phosphorylation deactivated Gsk-3 $\beta$, and subsequently inhibited Snail phosphorylation, leading to EMT (41). Kalra et al (42) reported that the knockdown of ILK suppressed p-Akt, p-Gsk-3 $\beta$, $\beta$-catenin, Snail, MMP-9 and Twist. We have demonstrated that the knockdown of ILK inhibited p-Akt and p-Gsk-3 $\beta$ although no significant changes were identified in Akt and Gsk-3 $\beta$ expression. The results also supported the above findings, indicating that ILK plays 
a vital role in the EMT process through the Akt/Gsk-3ß/Snail pathway, which was in agreement with the previous study (23). To investigate the effects of the ILK knockdown on the p-Akt and $\mathrm{p}-\mathrm{Gsk}-3 \beta$ induced by exogenous growth factors, HGF was applied prior to detection, which existed in the tumor microenvironment and activated PI3K. The results revealed that ILK was able to inhibit HGF-induced Akt and Gsk-3 $\beta$ phosphorylation to a certain extent.

In addition, the animal experiment showed that the knockdown of ILK significantly inhibited the tumor growth in xenograft models, with tumor inhibition rates of 55.56 or $53.57 \%$, respectively. ILK knockdown also suppressed spontaneous lymph node or distant metastasis in vivo. Compared with the control groups, the KD group exhibited lower microvascular density, which is necessary for tumor growth and metastasis. Cell proliferation and prevention of necrosis requires that tumor growth be dependent on adequate oxygen and nutrients, as induced by tumor angiogenesis (43). Immunohistochemical images of xenograft tumor tissue confirmed that ILK knockdown suppressed OSCC tumor growth, metastasis and EMT.

In conclusion, the findings of the present study have demonstrated that the knockdown of ILK inhibited the proliferation and metastasis of OSCC cells in vitro and in vivo. The present results suggest that ILK is crucial in cell progression, metastasis and EMT, by regulating key proteins such as Akt, Gsk-3 $\beta$, MMP-9, Snail, E-cadherin and caspase-3, which were essential for cell proliferation, apoptosis, metastasis, angiogenesis as well as EMT. Thus, ILK should be regarded as a newly proven oral cancer metastasis regulator and a potential therapeutic molecular target for OSCC.

\section{Acknowledgements}

This study was supported by grants from the Sichuan Science Foundation (contract grant nos. 2011SZ0156 and 2014SZ0204) and the National Natural Science Foundation of China (contract grant no. 81202134).

\section{References}

1. Scully C and Felix DH: Oral medicine - update for the dental practitioner oral cancer. Br Dent J 200: 13-17, 2006.

2. Bello IO, Soini Y and Salo T: Prognostic evaluation of oral tongue cancer: Means, markers and perspectives (II). Oral Oncol 46: 636-643, 2010

3. Hannigan GE, Leung-Hagesteijn C, Fitz-Gibbon L, Coppolino MG, Radeva G, Filmus J, Bell JC and Dedhar S: Regulation of cell adhesion and anchorage-dependent growth by a new beta 1-integrin-linked protein kinase. Nature 379: 91-96, 1996.

4. Zervas CG, Psarra E, Williams V, Solomon E, Vakaloglou KM and Brown NH: A central multifunctional role of integrin-linked kinase at muscle attachment sites. J Cell Sci 124: 1316-1327, 2011.

5. Hannigan G, Troussard AA and Dedhar S: Integrin-linked kinase: A cancer therapeutic target unique among its ILK. Nat Rev Cancer 5: 51-63, 2005.

6. Somasiri A, Howarth A, Goswami D, Dedhar S and Roskelley CD: Overexpression of the integrin-linked kinase mesenchymally transforms mammary epithelial cells. J Cell Sci 114: 1125-1136, 2001.

7. Wu C, Keightley SY, Leung-Hagesteijn C, Radeva G, Coppolino M, Goicoechea S, McDonald JA and Dedhar S: Integrin-linked protein kinase regulates fibronectin matrix assembly, E-cadherin expression, and tumorigenicity. J Biol Chem 273: 528-536, 1998
8. Nawshad A, Lagamba D, Polad A and Hay ED: Transforming growth factor- $\beta$ signaling during epithelial-mesenchymal transformation: Implications for embryogenesis and tumor metastasis. Cells Tissues Organs 179: 11-23, 2005.

9. Foroni C, Broggini M, Generali D and Damia G: Epithelial-mesenchymal transition and breast cancer: Role, molecular mechanisms and clinical impact. Cancer Treat Rev 38: 689-697, 2012.

10. Li R, Liu B, Yin H, Sun W, Yin J and Su Q: Overexpression of integrin-linked kinase (ILK) is associated with tumor progression and an unfavorable prognosis in patients with colorectal cancer. J Mol Histol 44: 183-189, 2013.

11. Li J, Yang ZL, Ren X, Zou Q, Yuan Y, Liang L, Chen M and Chen S: ILK and PRDX1 are prognostic markers in squamous cell/adenosquamous carcinomas and adenocarcinoma of gallbladder. Tumour Biol 34: 359-368, 2013.

12. Chen D, Zhang Y, Zhang X, Li J, Han B, Liu S, Wang L, Ling Y, Mao $S$ and Wang $X$ : Overexpression of integrin-linked kinase correlates with malignant phenotype in non-small cell lung cancer and promotes lung cancer cell invasion and migration via regulating epithelial-mesenchymal transition (EMT)-related genes. Acta Histochem 115: 128-136, 2013.

13. Watzka SB, Setinek U, Stubenberger EB, Tötsch M, Dekan G, Marcher M, Fleck T and Müller MR: Integrin-linked kinase, phosphorylated AKT and the prognosis of malignant pleural mesothelioma. Eur J Cardiothorac Surg 39: 180-184, 2011.

14. Serrano I, McDonald PC, Lock FE and Dedhar S: Role of the integrin-linked kinase (ILK)/Rictor complex in TGF $\beta$-1-induced epithelial-mesenchymal transition (EMT). Oncogene 32: 50-60, 2013.

15. Becker-Santos DD, Guo Y, Ghaffari M, Vickers ED, Lehman M, Altamirano-Dimas M, Oloumi A, Furukawa J, Sharma M, Wang Y, et al: Integrin-linked kinase as a target for ERG-mediated invasive properties in prostate cancer models. Carcinogenesis 33: 2558-2567, 2012.

16. Yan Z, Yin H, Wang R, Wu D, Sun W, Liu B and Su Q: Overexpression of integrin-linked kinase (ILK) promotes migration and invasion of colorectal cancer cells by inducing epithelial-mesenchymal transition via NF- $\mathrm{BB}$ signaling. Acta Histochem 116: 527-533, 2014.

17. Liang F, Zhang S, Wang B, Qiu J and Wang Y: Overexpression of integrin-linked kinase (ILK) promotes glioma cell invasion and migration and down-regulates E-cadherin via the NF- $\kappa \mathrm{B}$ pathway. J Mol Histol 45: 141-151, 2014.

18. Zhao D, Tang XF, Yang K, Liu JY and Ma XR: Over-expression of integrin-linked kinase correlates with aberrant expression of Snail, E-cadherin and N-cadherin in oral squamous cell carcinoma: Implications in tumor progression and metastasis. Clin Exp Metastasis 29: 957-969, 2012.

19. Zhao G, Guo LL, Xu JY, Yang H, Huang MX and Xiao G: Integrin-linked kinase in gastric cancer cell attachment, invasion and tumor growth. World J Gastroenterol 17: 3487-3496, 2011.

20. Gao J, Zhu J, Li HY, Pan XY, Jiang R and Chen JX: Small interfering RNA targeting integrin-linked kinase inhibited the growth and induced apoptosis in human bladder cancer cells. Int J Biochem Cell Biol 43: 1294-1304, 2011.

21. Liu Q, Xiao L, Yuan D, Shi X and Li P: Silencing of the integrin-linked kinase gene induces the apoptosis in ovarian carcinoma. J Recept Signal Transduct Res 32: 120-127, 2012.

22. Guo X, Wang W, Hu J, Feng K, Pan Y, Zhang L and Feng Y: Lentivirus-mediated RNAi knockdown of NUPR1 inhibits human nonsmall cell lung cancer growth in vitro and in vivo. Anat Rec (Hoboken) 295: 2114-2121, 2012.

23. Xing Y, Qi J, Deng S, Wang C, Zhang L and Chen J: Small interfering RNA targeting ILK inhibits metastasis in human tongue cancer cells through repression of epithelial-to-mesenchymal transition. Exp Cell Res 319: 2058-2072, 2013.

24. Maurizio Federico: Lentivirus Gene Engineering Protocols (Methods in Molecular Biology). Humana Press 229: 69-85, 2003.

25. Zheng YP, Liu H, Zeng H, Xiong L, Feng ZH and Sun NX: Downregulation of lentivirus-mediated ILK RNAi on tractional force generation in human retinal Müller cells. Acta Pharmacol Sin 30: 1625-1633, 2009.

26. Leong DT, Gupta A, Bai HF, Wan G, Yoong LF, Too HP, Chew FT and Hutmacher DW: Absolute quantification of gene expression in biomaterials research using real-time PCR. Biomaterials 28: 203-210, 2007.

27. Choi S and Myers JN: Molecular pathogenesis of oral squamous cell carcinoma: Implications for therapy. J Dent Res 87: 14-32, 2008 . 
28. Qiao B, Johnson NW and Gao J: Epithelial-mesenchymal transition in oral squamous cell carcinoma triggered by transforming growth factor- $\beta 1$ is Snail family-dependent and correlates with matrix metalloproteinase-2 and -9 expressions. Int J Oncol 37: $663-668,2010$

29. Voulgari A and Pintzas A: Epithelial-mesenchymal transition in cancer metastasis: Mechanisms, markers and strategies to overcome drug resistance in the clinic. Biochim Biophys Acta 1796: 75-90, 2009.

30. Araki K, Shimura T, Suzuki H, Tsutsumi S, Wada W, Yajima T, Kobayahi T, Kubo N and Kuwano H: E/N-cadherin switch mediates cancer progression via TGF- $\beta$-induced epithelial-to-mesenchymal transition in extrahepatic cholangiocarcinoma. Br J Cancer 105: 1885-1893, 2011.

31. Shin SY, Rath O, Zebisch A, Choo SM, Kolch W and Cho KH: Functional roles of multiple feedback loops in extracellular signal-regulated kinase and Wnt signaling pathways that regulate epithelial-mesenchymal transition. Cancer Res 70: 6715-6724, 2010.

32. Peinado H, Portillo F and Cano A: Transcriptional regulation of cadherins during development and carcinogenesis. Int J Dey Biol 48: 365-375, 2004.

33. Peinado H, Olmeda D and Cano A: Snail, Zeb and bHLH factors in tumour progression: An alliance against the epithelial phenotype? Nat Rev Cancer 7: 415-428, 2007

34. Radeva G, Petrocelli T, Behrend E, Leung-Hagesteijn C, Filmus J, Slingerland J and Dedhar S: Overexpression of the integrin-linked kinase promotes anchorage-independent cell cycle progression. J Biol Chem 272: 13937-13944, 1997.

35. Persad S, Attwell S, Gray V, Delcommenne M, Troussard A, Sanghera $\mathbf{J}$ and Dedhar S: Inhibition of integrin-linked kinase (ILK) suppresses activation of protein kinase B/Akt and induces cell cycle arrest and apoptosis of PTEN-mutant prostate cancer cells. Proc Natl Acad Sci USA 97: 3207-3212, 2000.
36. D'Amico M, Hulit J, Amanatullah DF, Zafonte BT, Albanese C, Bouzahzah B, Fu M, Augenlicht LH, Donehower LA, Takemaru K, et al: The integrin-linked kinase regulates the cyclin D1 gene through glycogen synthase kinase 3beta and cAMP-responsive element-binding protein-dependent pathways J Biol Chem 275: 32649-32657, 2000.

37. Fielding AB, Dobreva I, McDonald PC, Foster LJ and Dedhar S: Integrin-linked kinase localizes to the centrosome and regulates mitotic spindle organization. J Cell Biol 180: 681-689, 2008.

38. Fielding AB, Lim S, Montgomery K, Dobreva I and Dedhar S: A critical role of integrin-linked kinase, ch-TOG and TACC3 in centrosome clustering in cancer cells. Oncogene 30: 521-534, 2011.

39. Fielding $A B$ and Dedhar S: The mitotic functions of integrin-linked kinase. Cancer Metastasis Rev 28: 99-111, 2009.

40. Koul D, Shen R, Bergh S, Lu Y, de Groot JF, Liu TJ, Mills GB and Yung WK: Targeting integrin-linked kinase inhibits Akt signaling pathways and decreases tumor progression of human glioblastoma. Mol Cancer Ther 4: 1681-1688, 2005.

41. Doble BW and Woodgett JR: Role of glycogen synthase kinase-3 in cell fate and epithelial-mesenchymal transitions. Cells Tissues Organs 185: 73-84, 2007.

42. Kalra J, Sutherland BW, Stratford AL, Dragowska W, Gelmon KA, Dedhar S, Dunn SE and Bally MB: Suppression of Her2/neu expression through ILK inhibition is regulated by a pathway involving TWIST and YB-1. Oncogene 29: 6343-6356, 2010.

43. Muramatsu M, Yamamoto S, Osawa T and Shibuya M: Vascular endothelial growth factor receptor-1 signaling promotes mobilization of macrophage lineage cells from bone marrow and stimulates solid tumor growth. Cancer Res 70: 8211-8221, 2010. 